\title{
PERCEPCIÓN DEL PROCESO SALUD-ENFERMEDAD Y DEMANDA DEL CUIDADO EN COMUNIDADES RURALES DE CHIMBOTE-PERÚ
}

\author{
Juan Miguel Ponce Loza', Esperanza Melgarejo Valverde², Yolanda Rodriguez Barreto ${ }^{3}$, Diana Guibovich Arroyo ${ }^{4}$
}

Objetivo de conocer la relación entre percepción del proceso salud-enfermedad con las demandas de cuidado de la salud en las comunidades rurales del distrito de Chimbote. La población estuvo conformada por 88 pobladores. Para la recolección de datos se utilizó la técnica de encuesta. El riesgo relativo (Odd Ratio) y chi cuadrada (x2) se utilizaron para el análisis de relaciones entre percepción y demanda del cuidado. Resultados: La mayoría tiene una percepción biológica del proceso salud-enfermedad (59.1\%), seguido de la ecológica (35.2\%) e, histórico social (5.7\%). Prevalece una demanda de cuidado clínico asistencial (49.9\%), autoatención (38.6\%) y, lo espiritual 20.5\%. La percepción del proceso salud-enfermedad se relaciona significativamente con las demandas de cuidado de la salud ( $p=0,0245)$, y existe 3 veces más riesgo que los pobladores demanden un cuidado clínico asistencial si la percepción es biológica $(\mathrm{OR}=3.2)$. Los pobladores perciben el proceso salud-enfermedad como producto de variables biológicas, ecológicas e histórico sociales, conllevándoles a prácticas de la salud alternativa y la salud tradicional. Descriptores: Salud, Enfermedad, Atención a la Salud, Enfermería en Salud Comunitaria.

\section{PERCEPTION OF THE PROCESS HEALTH - DISEASE AND THE DEMANDS OF HEALTH CARE IN THE RURAL COMMUNITIES OF CHIMBOTE -PERU.}

This motivated to realize the investigation with the aim to know the relation between perception of the process health - disease with the demands of care of the health in the rural communities of Chimbote district. The population was shaped by 88 settlers. For the compilation of information the technology of survey was in use. The relative risk (Odd Ratio) and chi squared (x2) they were in use for the analysis of relations between perception and demand of the care. Results: The majority has a biological perception of the process health - disease (59.1\%), followed by the ecological one (35.2 \%) and, historically socially (5.7\%). There prevails a demand of clinical welfare care (49.9\%), autoattention (38.6\%) and, the spiritual thing $20.5 \%$. The perception of the process health - disease relates significantly to the demands of care of the health $(p=0,0245)$, and there exists 3 times more risk than the settlers demand a clinical welfare care if the perception is biological $(\mathrm{OR}=3.2)$. The settlers perceive the process health - disease as product and historical of biological, ecological social variables, carrying them to practices of the alternative health and the traditional health.

Descriptors: Health, Disease, Health Care, Infirmary in Community Health.

\section{PERCEPÇÃO DO PROCESSO SAÚDE-DOENÇA E DEMANDA DE CUIDADOS EM COMUNIDADE RURAIS DE CHIMBOTE-PERU}

Objetivo: conhecer a relação entre a percepção do processo saúde-doença com as demandas de cuidados de saúde em comunidades rurais do distrito de Chimbote. Trata-se de uma pesquisa quantitativa que utilizou um survey para a coleta de dados. A população foi composta por 88 moradores. O risco relativo (OddsRatio) e qui-quadrado (x2) foram utilizados para analisar as relações entre percepção e demanda de da procura de cuidados. Resultados: A maioria tem uma percepção biológica do processo saúde-doença (59,1\%), seguida da ecológica (35,2\%) e histórico-social (5,7\%). Prevalece uma demanda de cuidado clínico-assistencial (49,9\%), autocuidado (38,6\%) e espiritual (20,5\%). A percepção do processo saúde-doença se relaciona significativamente com as demandas de cuidados de saúde ( $p=0,0245)$, existindo 3 vezes maior risco que os moradores exijam um cuidado clínico se sua percepção é biológico (OR = 3.2). Os moradores percebem o processo saúde-doença como produto de variáveis biológicas, ecológicas e históricas, conduzindo-os a práticas de saúde alternativa e saúde tradicional.

Descritores: Saúde, Doença, Cuidados de Saúde, Saúde Comunitária.

${ }^{1}$ Doctor en Salud Pública. Universidad Nacional del Santa. Email: jmikeponce@hotmail.com 2Magister en Administración de Salud. Universidad del Santa

${ }^{3}$ Maestra en Enfermaria. Universidad del Santa

${ }^{4}$ Maestra en Ciencias de Enfermería. Universidad del Santa. 


\section{INTRODUCCIÓN}

El concepto de salud han evolucionado a lo largo del tiempo, tanto en su concepción como en su práctica ${ }^{(1,2,3)}$. Nuestras instituciones de salud son bastante homogéneas en cuanto al modo de producir servicios, forzando la adaptación de la población a su funcionamiento ${ }^{(4.5)}$. En tal sentido, desde la gestión de los servicios se torna prioritario modificar las prácticas de los equipos, tomándolas menos rígidas y paternalistas ${ }^{(5,6,67)}$.

En lo que refiere a la población de estudio, interesa, indagar sobre el proceso que va desde la percepción de las necesidades de cuidado de la salud hasta su efectivización en demanda y posterior utilización, donde el proceso de atención surge como respuesta a una necesidad (8,9,10)

Debe tenerse en cuenta que la percepción de necesidad y su jerarquización depende de aquél que la percibe y de la concepción propia del proceso de salud-enfermedad, aspectos que condicionarán la modalidad que adopte la demanda, que es en síntesis, la necesidad manifestada por la persona que la percibió ${ }^{(11.12)}$. En Brasil, estudio refiere que, los niños, mujeres en edad fértil, ancianos, la clase social baja y el grupo con menos educación se asociaron con la utilización de servicios de salud ${ }^{(13)}$. En Costa Rica, otro estudio concluye que los factores determinantes de la utilización de consultas médicas fueron el nivel educativo, el estado de salud percibida, el número de enfermedades crónicas declaradas y la región de residencia ${ }^{(14)}$.

En Argentina, en un abordaje cualitativo con familias de diferentes sectores sociales y áreas geográficas, concluye que existen modalidades diferenciadas de percibir la enfermedad y consiguientemente, comportamientos diferenciales en lo que hace a búsqueda de atención, elección del tipo de efector de salud al que concurrir, prácticas preventivas y de cuidado, etc ${ }^{(15)}$. Asimismo, la accesibilidad al sistema de salud es uno de los principios fundamentales de la Atención Primaria de la Salud (APS) ${ }^{(16)}$

En Perú, las concepciones, actitudes y prácticas de salud son parcialmente adecuadas ${ }^{(17)}$. Este problema afecta a las familias de diferentes estratos sociales, evidenciándo se mayormente en las comunidades rurales que urbanas y urbano marginales, como es el caso de la comunidad rural "San Jóse" y, la comunidad rural "Santa Elisa" del distrito de Chimbote ${ }^{(12)}$.
Las Comunidades "San José" y "Santa Elisa", se encuentra ubicada en una zona rural, perteneciente al Distrito de Chimbote, presentando una población aproximada de 750 habitantes entre ambas, que conforman aproximadamente 140 familias, las viviendas están distribuidas por lotes. Con un gobierno democrático presidido por un secretario general, además cuenta con un teniente gobernador y agente municipal. Se observa la presencia de un Institución Educativa de Nivel Primario, un PRONOE y Vaso de Leche. La mayoria de la población tiene grado de instrucción primaria, la población refiere tener la religión católica, pero practican mas la evangélica, el principal sustento económico de sus pobladores se basa en la agricultura, compuesta generalmente por familias extensas, donde el soporte económico es brindado por el padre de familia y la madre es responsable de las labores doméstica y crianza de los hijos. Respecto al saneamiento ambiental no tienen servicios de aqua potable ni desagüe, eliminando sus excretas en letrinas, la eliminación de basura es a campo abierto y presentan servicios de alumbrado público y domiciliario. El transporte para la ciudad de Chimbote no es continuo, agudizándose la situación en situaciones de emergencias por las noches. En este contexto los pobladores de las comunidades rurales tienen su propia dinámica social, y visión de la salud enfermedad así como para enfrentarla ${ }^{(12)}$.

Por lo expuesto y conocedores del importante rol de enfermería en la intervención en el proceso salud-enfermedad, nos planteamos la siguiente interrogante:

¿Cómo se relaciona la percepción del proceso saludenfermedad con las demandas de cuidado de la salud en las comunidades rurales del Distrito de Chimbote?

\section{Objetivo General:}

Conocer la relación entre Percepción del proceso saludenfermedad con las demandas de cuidado de la salud en las comunidades rurales del distrito de Chimbote.

\section{Objetivos Específicos:}

a. Identificar el nivel de Percepción del proceso saludenfermedad en las comunidades rurales del distrito de Chimbote. 
b. Identificar las demandas de cuidado de la salud en las comunidades rurales del distrito de Chimbote.

c. Determinar la relación entre Percepción del proceso salud-enfermedad y las demandas de cuidado de la salud en las comunidades rurales del distrito de Chimbote.

\section{Material y método}

La investigación es de tipo cuantitativa, descriptiva correlacional de corte transversal, desarrollado en las comunidades rurales "Santa Elisa" y "San José" del distrito de Chimbote, de la provincia del Santa, Región Ancash.

La población de estudio está constituida por 88 pobladores, de los cuales 37 pobladores de "Santa Elisa" y 51 pobladores de "San José".

Para efecto de la investigación se considera percepción del proceso salud-enfermedad, a la expresión y entendimiento de la salud y de la enfermedad, como producto de su cultura, que oriente al sujeto o sociedad hacia algún enfoque del proceso-salud enfermedad. Sus categorías son: percepción biológica, ecológica, e histórica social(18). Y demandas de cuidado de la salud, en la medida de su preocupación, interés, afecto, importarse o protegerse, acuden a diversas instancias para atender sus necesidades y/o problemas de salud, así consideramos una demanda clínica asistencial, demanda espiritual y demanda autoatención ${ }^{(19)}$. Como instrumentos de recolección de datos: Cuestionario de Percepción del proceso salud-enfermedad y de demandas de cuidado de la salud. Los datos fueron procesados usando el software estadístico Epiinfo v. 3.4.3. El análisis estadístico se realizó a nivel descriptivo y analítico.

\section{RESULTADOS Y DISCUSIÓN}

Sobre la percepción del proceso salud-enfermedad en las comunidades rurales del Distrito de Chimbote, se observa que la mayoría de pobladores tienen una percepción biológica (59.1\%), seguido de la percepción ecológica (35.2\%) y, con mínima proporción la percepción histórico social (5.7\%). La alta prevalencia de percepción biológica del proceso salud enfermedad, expresa el énfasis que los pobladores remarcan que la enfermedad es producto de un agente externo de tipo biológico.

La tercera parte de la población en estudio tiene una percepción ecológica del proceso salud enfermedad, ampliando los pobladores su entendimiento sobre las causas de la enfermedad, este grupo no solo considera una causa de la enfermedad como el enfoque biológico (agente etiológicoenfermedad), sino que entiende que las enfermedades están asociadas a diversos factores químicos, físicos, ambientales, socioculturales, muchos de ellos relacionados con el modo de vida y el trabajo. La enfermedad es la resultante del desequilibrio entre tres factores, agente causal, huésped y medio ambiente. El enfoque ecológico considera los aspectos culturales y sociales pero de una manera superficial sin fundamentar la explicación de éste en el proceso Salud-Enfermedad, solamente se basa en la adaptación y desadaptación, es decir mantener que el sistema funcione ${ }^{(17)}$.

Los resultados muestran una mínima proporción de pobladores con percepción histórica social, es la población que va más allá de la forma de vida, son los que consideran componentes mas estructurales, como la dimensión económica, política, cultural, ambiental, como explicación de fondo de las enfermedades.

En contraste con el sector salud, que ejerce en los hospitales un enfoque puramente clínico-biologista; cuando las causas de la enfermedad deberían buscarse no solamente en los procesos biológicos o en las características de la triada ecológica (huésped, agente, ambiente); sino en los procesos sociales, en la producción y la reproducción social (17)

Sobre las demandas de cuidado de la salud en las comunidades rurales del Distrito de Chimbote, se observa que la mayoría de pobladores tienen una demanda de cuidado clínico asistencial (49.9\%), seguido de la autoatención (38.6\%) $y$, una quinta parte de los pobladores demanda lo espiritual (20.5\%).

Aproximadamente la mitad de los pobladores demandan cuidado clínico asistencial, es decir los pobladores expresan su tendencia a enfocar la solución a la enfermedad (inhibir o aniquilar al agente patológico), y pierde el contexto del enfermo (persona), esto le conlleva a utilizar servicios que oferten servicios de 
laboratorioy, de anatomía patológica. Un ejemplo son los servicios de hospitalización, del centro de salud y del puesto de salud.

La autoatención es otra opción de los pobladores para resolver sus problemas de salud, y representa más de la tercera parte de la población en estudio. Esta demanda se explica porque el financiamiento de la salud que debería asumirlo el Estado ahora lo está cargando al presupuesto familiar, los costos de los servicios y programas de salud. En tal sentido los pobladores evitan pagar la consulta médica o clínica, y acuden a medicinas alternativas y tradicionales ${ }^{(8,9)}$.

Es preciso remarcar que una quinta parte de la población busca solucionar sus problemas de salud a través de lo espiritual. Esta proporción de pobladores generalmente pertenecen y practican activamente dentro de una iglesia evangélica, que cada día aumenta significativamente en nuestra sociedad. La iglesia trata todas aquellas patologías en las que la medicina no tiene eficacia de curación tales como las enfermedades terminales o sociales (cáncer, adicciones, VIH...) y curarse a través de la fe es el principal recurso ${ }^{(15)}$

Respecto a la relación entre percepción del proceso salud-enfermedad y las demandas de cuidado de la salud en las comunidades rurales del Distrito de Chimbote (Tabla 1), se observa que entre los pobladores con percepción biológica del proceso salud-enfermedad prevalece la demanda de cuidado clínico asistencial (51.90\%), a diferencia de los pobladores con percepción ecológica del proceso salud-enfermedad en quienes prevalece la demanda de cuidado autoatención y espiritual (38.7\% y $35.5 \%$ respectivamente), y a diferencia de los pobladores con percepción histórico social del proceso-salud enfermedad, en quienes la demanda de cuidado espiritual y autoatención (40.0\% y 40.0\% respetivamente). Existe relación significativa entre la percepción del proceso salud-enfermedad y las demandas de cuidado de la salud $(p=0,0245)$. Ante la prueba de riesgo, el tener una percepción biológica del proceso salud enfermedad se constituye en riesgo elevado para una demanda de cuidado de la salud clínico asistencial (OR=3.2), es decir, existe 3.2 veces más riesgo que los pobladores demanden un cuidado de la salud clínico asistencial si la percepción del proceso salud enfermedad es biológica.

Los resultados expresan coherencia entre la percepción del proceso salud-enfermedad y la demanda del cuidado de la salud, observándose predominio de una percepción biológica y una demanda clínica asistencial, preocupándose mas por la enfermedad que por la persona enferma, considera a la enfermedad como dolencia que el médico puede reconocer, diagnosticar, clasificar y curar. El cuerpo del ser humano se concibe como una máquina, la enfermedad como un daño a la máquina y el médico el mecánico que la repara $(16.17)$

Este enfoque deja afuera todo lo relacionado con la esfera psicosocial. La cultura, la religión los hábitos no tendrían participación en el proceso de salud enfermedad (12).

En contraste entre los pobladores con percepción ecológica e histórico social del proceso salud-enfermedad prevalece la demanda de cuidado autoatención y espiritual, percibiendo que la salud-enfermedad es producto de variables tanto biológicas, sociales y culturales, conllevándoles a prácticas de la salud alternativa y la salud tradicional, que tiene una base en su propia cultura ${ }^{(12)}$.

Tabla 1 - Percepción del proceso salud-enfermedad y las demandas de cuidado de la salud en las comunidades rurales del distrito de Chimbote

\begin{tabular}{|c|c|c|c|c|c|c|c|c|}
\hline \multirow{3}{*}{$\begin{array}{l}\text { PERCEPCIÓN DEL PROCESO } \\
\text { SALUD-ENFERMEDAD }\end{array}$} & \multicolumn{8}{|c|}{ DEMANDAS DE CUIDADO DE LA SALUD } \\
\hline & \multicolumn{2}{|c|}{${ }^{*}$ Clínico Asistencial } & \multicolumn{2}{|c|}{ Espiritual } & \multicolumn{2}{|c|}{ Autoaten-ción } & \multicolumn{2}{|c|}{ TOTAL } \\
\hline & $\mathrm{fi}$ & hi & $\mathrm{fi}$ & hi & $\mathrm{fi}$ & hi & $\mathrm{fi}$ & hi \\
\hline *Biológica & 27 & 51,9 & 5 & 9,6 & 20 & 38,5 & 52 & 59,1 \\
\hline Ecológica & 8 & 25,8 & 11 & 35,5 & 12 & 38,7 & 31 & 35,2 \\
\hline Histórico social & 1 & 20,0 & 2 & 40,0 & 2 & 40,0 & 5 & 5,7 \\
\hline TOTAL & 36 & 40,9 & 18 & 20,5 & 34 & 38,6 & 88 & 100.0 \\
\hline \multicolumn{9}{|c|}{$\begin{array}{c}X 2=11.1933 \mathrm{gl}=4 \quad \mathrm{p}=0.0245 \text { Significativo } \\
{ }^{*} \mathrm{OR}=3.2 \text { Riesgo elevado }\end{array}$} \\
\hline
\end{tabular}




\section{CONCLUSIONES}

La comunidad rural tiene su propia dinámica social y percepción del proceso salud-enfermedad que se relaciona con las demandas de cuidado de salud, resolviendo cotidianamente cómo cuidar la salud y cómo recuperarla cuando se ha deteriorado.

\section{REFERÊNCIAS}

1. Vergara, Q. MC. Representaciones sociales que orientan la experiencia de vida de algunos grupos de jóvenes de la ciudad de Manizales. [Tesis doctoral]. Manizales: Universidad de Manizales; 2006.

2. Suárez, R. Salud-enfermedad: una categoría a repensar desde la antropología. En R. Suárez (comp.), Reflexiones en salud: una aproximación desde la antropología. Bogotá: Universidad de los Andes, 2001.

3. Mariano, H; Ramos, M. y Fernández. A. Salud y juventud. Edita. Consejo de la juventud de España. 2001ISBN. B4-921107-9-1.

4. Kornblit, L. y Mendes, A. La Salud y la Enfermedad: Aspectos biológicos y sociales. contenidos curriculares. México; 2008.

5. Dávila, M. Perú: Estudio de demanda de Servicios de Salud (Documento 1). Informe Técnico No. 012. Bethesda, MD: Socios para la reforma de sector salud. Abt Associates Inc. Perú: 2002.

6. Sociedad Española de Médicos en Atención Primaria. Atención Primaria: Pasado presente y futuro. SEMERGENIE Business School. España; 2009.

7. Merzel, C. Gender differences in health care access indicators in an urban, low-income community. American Journal of Public Health, Washington; 2000

8. Mechanic, D. Public Expectations and Health Care: Essays on the changing Organization of Health Services; 2008. Citado en Aday, A. Andersen, R. Marco teórico para el estudio del acceso a la atención médica. Investigaciones sobre servicios de salud. Publicación científica №534. OPS, Washington; 1992.

9. Kohn y White, citado en Navas-Moiso. Modelos conceptuales para la investigación de necesidades en salud y utilización de servicios en poblaciones localizadas. Cuadernos Médico Sociales $N^{\circ} 69,1994$. Argentina; 1994
10. White, K. Williams F., Greenberg B. La ecología de la atención médica. Investigaciones sobre servicios de salud. Publicación científica N ${ }^{\circ} 534$ OPS. 1992. Washington; 1992.

11. Boltanski, L. Los usos sociales del cuerpo. Ed. Periferia, Buenos Aires: 1995

12. Ponce, M. Intervención de Enfermería en Salud Comunitaria-IESCO Documento de trabajo; Sección de Salud Comunitaria, EAPE-FC-UNS Nuevo Chimbote-Perú; 2004

13. Mendoza-Sassi, R. y Béria, J. Utilización de los servicios de salud: una revisión sistemática sobre los factores relacionados. Brasil; 2002.

14. Morera, M. y Aparicio, A. Determinantes de la utilización de servicios de salud en Costa Rica. Gaceta Sanitaria, Volume 24, Issue 5, Pages 410415. Costa Rica; 2009

15. Díaz, A. y otros. La dimensión sociocultural y su relación con los patrones de utilización acerca de un centro de salud de atención primaria desde la perspectiva de la población. Argentina; 2009.

16. Landini, F. et al. Problemas y propuestas para la conceptualización de la accesibilidad psicosocial y cultural al sistema de salud Argentina. Argentina; 2011

17. Vela, A. y otros. Concepciones, actitudes y prácticas en salud de la población distrito de Yarabamba, Arequipa. Perú; 2009.

18. Breilh, J y Granda, E. Investigación de Salud en la Sociedad CEASQuito. Ecuador; 1994

19. Vergara, M. Tres concepciones históricas del proceso saludenfermedad. [Tesis doctoral]. Manizales, Facultad de Ciencias Sociales. Universidad Autónoma de Manizales. Colombia. 2007.

Recebido: 24/09/2013

Aceito: 09/12/2015 